\title{
Comportamento ingestivo de ovinos Santa Inês alimentados com dietas contendo farelo de cacau ${ }^{1}$
}

\section{Gleidson Giordano Pinto de Carvalho², Aureliano José Vieira Pires ${ }^{3,6}$, Robério Rodrigues Silva ${ }^{4}$, Leandro Sampaio Oliveira Ribeiro ${ }^{5}$, Daiane Maria Trindade Chagas $^{5}$}

\author{
${ }^{1}$ Projeto financiado pela UESB, Itapetinga, BA. \\ 2 Doutorando em Zootecnia, UFV, Viçosa, MG. Bolsista do CNPq. \\ ${ }^{3}$ Departamento de Tecnologia Rural e Animal, UESB, Itapetinga, BA. \\ ${ }^{4}$ Doutorando em Zootecnia, UEM, Maringá, PR. \\ ${ }^{5}$ Graduando em Zootecnia, UESB, Itapetinga, BA. Bolsista de Iniciação Científica (CNPq e FAPESB). \\ ${ }^{6}$ Pesquisador do CNPq.
}

RESUMO - O experimento foi conduzido para avaliar o comportamento ingestivo de ovinos alimentados com dietas contendo farelo de cacau. Foram utilizados 16 ovinos Santa Inês fêmeas, não-gestantes e não-lactantes, com peso corporal médio de $25 \mathrm{~kg}$ e aproximadamente 12 meses de idade, mantidos em baias individuais. O farelo de cacau foi fornecido no concentrado nos níveis de $0,10,20$ e $30 \%$ e, como volumoso, utilizou-se feno de mandioca. As dietas foram fornecidas em mistura completa, na proporção 50:50 volumoso:concentrado. Os tempos de alimentação, ruminação e ócio obtidos em 24 horas de observação foram semelhantes. Contudo, os animais que consumiram dietas com maiores níveis de farelo de cacau, acima de 14,8\% de substituição no concentrado, reduziram o número de bolos ruminados por dia, que foi compensado pelo aumento do tempo de mastigações por bolo. Os consumos de matéria seca (MS) e fibra em detergente neutro (FDN) e a eficiência de alimentação (g MS e FDN/hora) não foram influenciados pelos níveis de farelo de cacau na dieta, entretanto, esse alimento provocou alterações na eficiência de ruminação (g MS e FDN/bolo). O número de mastigações merícicas por bolo ruminado aumentou linearmente, enquanto o número de mastigações por dia apresentou comportamento quadrático, com valor máximo de 42.818,4 mastigações diárias para o nível de 16,9\% de farelo de cacau. Embora não se tenha verificado diferença nos consumos de MS e FDN (kg/dia), a inclusão de farelo de cacau em dietas para ovinos Santa Inês afetou alguns parâmetros do comportamento ingestivo.

Palavras-chave: ócio, ruminação, tempo de alimentação

\section{Ingestive behavior of Santa Inês sheep fed diets with cocoa meal}

\begin{abstract}
The experiment was carried out to evaluate the ingestive behavior of sheep fed diets containing cocoa meal. Sixteen Santa Inês female sheep, no-pregnant, no-lactating, averaging $25 \mathrm{~kg}$ of body weight and 12 months of age, maintained in individual barns were used. The cocoa meal was fed in the concentrate at the levels of $0,10,20$ and $30 \%$, and as forage was cassava aerial part hay. The diets were offered in total mixed ration, in 50:50 forage:concentrate ratio. Feeding, ruminating and idle times obtained in 24 hours of observation were similar; however, animals that consumed diets with higher cocoa meal levels, over $14.8 \%$ of substitution in the concentrate, reduced the number of ruminated boli per day, which was balanced by the increase of chewing time per bolus. Dry matter (DM) intake, neutral detergent fiber (NDF) intake and feeding efficiency (g DM and NDF/hours) were not influenced by cocoa meal levels of animal diets. However, this feed promoted alterations in rumination efficiency (g DM and NDF/bolus). The ruminating chewing number per ruminated bolus linearly increased while chewing number per day showed quadratic behavior, with maximum estimated value of 42,818.4 daily chewing for $16.9 \%$ cocoa meal level. Although no difference was observed for DM and NDF (kg/day) intake, cocoa meal inclusion to Santa Inês sheep diet affected some ingestive behavior parameters.
\end{abstract}

Key Words: feeding, time, idle, rumination

\section{Introdução}

O estudo do comportamento ingestivo tem recebido atenção crescente de pesquisadores das áreas de Produção e Nutrição Animal (Miranda et al., 1999; Burguer et al., 2000; Carvalho et al., 2004; Silva et al., 2004, 2005a; Carvalho et al., 2006a; Carvalho et al., 2007). A intensificação do processo de domesticação animal e, posteriormente, do processo 
produtivo levou a grandes concentrações de animais em áreas cada vez mais restritas. Além disso, os problemas decorrentes da sazonalidade de produção de forragem no Brasil, associados à crescente demanda de produtos de origem animal, têm aumentado o número de animais confinados em todo o País.

Animais confinados normalmente consomem elevada quantidade de concentrados para suprir a demanda energética e protéica para mantença e produção. Entre os alimentos concentrados mais utilizados na alimentação animal, tanto de ruminantes como de monogástricos, destacam-se o milho e o farelo de soja, pois ambos formam uma excelente combinação de energia (milho) e proteína (farelo de soja) de alto valor biológico. Contudo, o elevado custo destes alimentos constitui fator limitante à sua utilização.

Neste contexto, esforços têm sido despendidos na busca por alimentos alternativos de baixo custo que possam substituir parcial ou totalmente os alimentos concentrados como o milho e o farelo de soja, de elevado custo (Pires et al., 2004, 2005; Silva et al., 2005b). Em estudos recentes, foi demonstrado que o farelo de cacau pode ser utilizado com sucesso na alimentação de ruminantes (Cunha Neto, 2004; Pires et al., 2004; Pires et al., 2005). Contudo, Carvalho et al. (2006a) alertaram que as condições de alimentação podem modificar os parâmetros do comportamento ingestivo, uma vez que as propriedades físicas e químicas dos subprodutos diferem das plantas forrageiras.

Desse modo, conduziu-se este trabalho para avaliar o comportamento ingestivo de ovinos Santa Inês alimentados com dietas contendo farelo de cacau.

\section{Material e Métodos}

O experimento foi desenvolvido na Universidade Estadual do Sudoeste da Bahia, no Setor de Ovinocultura e no Laboratório de Forragicultura e Pastagem do Departamento de Tecnologia Rural e Animal.

Foram utilizados 16 ovinos Santa Inês fêmeas, não-prenhes e não-lactantes, com peso vivo médio inicial de $25 \mathrm{~kg}$ e média de 12 meses de idade. Os animais foram alojados em baias individuais de 1,2 $\mathrm{m}^{2}$, com divisórias em grades de madeira, providas de cocho e bebedouro individuais, e com piso de alvenaria. No início do experimento, os animais foram vermifugados, pesados, identificados com plaquetas fixas a colares e distribuídos por sorteio nos tratamentos.

Os animais foram alimentados com dietas isoprotéicas (16\% de proteína), em mistura completa, uma vez por dia, pela manhã, e receberam água ad libitum. As dietas foram compostas de volumoso, concentrado, farelo de cacau e sal mineral. Como volumoso, utilizou-se feno da parte aérea de mandioca (PAM), triturado em moinho desintegrador, na proporção de 50:50\% volumoso e concentrado. O concentrado, à base de milho e farelo de soja, foi substituído em 0 , 10,20 e $30 \%$ pelo farelo de cacau, que correspondeu a 0,5 , 10 e $15 \%$ da dieta total.

As dietas foram fornecidas em quantidade ajustada para ocorrer $10 \%$ de sobras. Semanalmente, foram coletadas as sobras e amostras de alimentos para análises laboratoriais.

Os teores de proteína bruta (PB), extrato etéreo (EE), fibra em detergente neutro (FDN), fibra em detergente ácido (FDA) e proteína insolúvel em detergente neutro (PIDN) das dietas experimentais (Tabela 1 ) foram obtidos conforme metodologia descrita por Silva \& Queiroz (2002).

As porcentagens de carboidratos não-fibrosos (CNF) e totais (CT) foi obtida pelas equações propostas por Sniffen et al. (1992): CNF $=(100-\% F D N c p-\% P B-\% E E$ - \%cinzas) e CT $=100-(\% \mathrm{~PB}+\% \mathrm{EE}+\%$ cinzas $)$.

O experimento teve duração total de 78 dias: os primeiros 15 dias foram destinados à adaptação dos animais às dietas e ao ambiente e os demais foram divididos em três períodos de 21 dias (fase de coleta de dados). O comportamento alimentar dos animais foi determinado nos dois últimos dias do período experimental pela quantificação dos intervalos de tempo durante 24 horas/ dia (Fischer, 1996).

No registro do tempo despendido em alimentação, ruminação e ócio, adotou-se a observação visual dos animais a cada 10 minutos, feita por quatro observadores treinados, em sistema de revezamento, posicionados estrategicamente de forma a não incomodar os animais, o que totalizou 288 observações por período. No mesmo dia, foi realizada a contagem do número de mastigações merícicas $\mathrm{MM}_{\mathrm{nb}}$ (no/bolo) e do tempo despendido para ruminação de

Tabela 1 - Composição nutricional das dietas

\begin{tabular}{lrrrr}
\hline \multirow{2}{*}{ Item } & \multicolumn{3}{c}{ Farelo de cacau no } & \multicolumn{2}{c}{ concentrado (\%) } \\
\cline { 2 - 5 } & \multicolumn{1}{c}{0} & 10 & \multicolumn{1}{c}{20} & \multicolumn{1}{c}{30} \\
\hline Proteína bruta $^{1}$ & 15,98 & 16,00 & 16,01 & 15,97 \\
Extrato etéreo $^{1}$ & 3,29 & 3,87 & 4,26 & 4,89 \\
Fibra em detergente neutro $^{1}$ & 34,22 & 38,21 & 40,66 & 41,56 \\
Fibra em detergente ácido $^{1}$ & 15,13 & 17,56 & 19,12 & 20,93 \\
Hemicelulose $^{1}$ & 18,09 & 20,65 & 21,54 & 20,63 \\
Carboidratos totais $^{1}$ & 75,12 & 75,54, & 75,88 & 76,69 \\
Carboidratos não-fibrosos $^{1}$ & 48,93 & 46,78 & 44,36 & 41,72 \\
Lignina $^{1}$ & 5,63 & 6,21 & 6,98 & 7,12 \\
Proteína insolúvel $_{\text {em detergente neutro }}{ }^{2}$ & 11,09 & 13,25 & 19,78 & 24,41 \\
\hline
\end{tabular}

${ }^{1}$ em $\%$ da MS; ${ }^{2}$ em $\%$ da PB. 
cada bolo $\mathrm{MM}_{\mathrm{tb}}$ (seg/bolo) utilizando-se um cronômetro digital. Para a obtenção das médias das mastigações e do tempo, foram feitas as observações de três bolos ruminais em três períodos diferentes do dia (10-12, 14-16 e 18-20 horas). Foram computados o tempo e o número de mastigações para cada bolo ruminal por animal.

As variáveis g de MS e FDN/bolo foram obtidas dividindo-se o consumo médio individual de cada fração pelo número de bolos ruminados por dia (em 24 horas). Para obtenção do número de bolos diários, procedeu-se à divisão do tempo total de ruminação pelo tempo médio gasto na ruminação de cada bolo, descrito anteriormente. A eficiência de alimentação e ruminação, expressa em g MS/hora e g FDN/hora, foi obtida pela divisão do consumo médio diário de MS e FDN pelo tempo total despendido em alimentação e/ou ruminação em 24 horas, respectivamente. Essas e outras variáveis obtidas neste experimento, como o número de bolos ruminais por dia (NBR), o tempo de mastigação total (TMT) e o número de mastigações merícicas por dia $\left(\mathrm{MM}_{\mathrm{nd}}\right)$, foram obtidas conforme metodologia descrita por Bürger et al. (2000) e Polli et al. (1996). Durante a coleta de dados, na observação noturna dos animais, o ambiente foi mantido com iluminação artificial.

O experimento foi analisado segundo um delineamento inteiramente casualizado, com quatro repetições para cada tratamento. Os resultados foram submetidos à análise de variância e, posteriormente, à decomposição ortogonal da soma de quadrados de tratamentos em efeitos de ordem linear, quadrática, cúbica e quártica, com posterior ajuste de regressões lineares. Todos os procedimentos estatísticos foram realizados utilizando-se o programa SAEG - Sistema de Análises Estatísticas e Genéticas (UFV, 2000) e nível crítico de probabilidade de 10\%.

\section{Resultados e Discussão}

O tempo despendido nas atividades de alimentação, ruminação e ócio em 24 horas não foi afetado pela adição de farelo de cacau na dieta dos animais; os valores médios registrados foram, respectivamente, de 301,88; 466,88 e 671,25 minutos (Tabela 2). O fato de as dietas terem sido isoprotéicas e apresentado pequenas variações nos teores de fibra pode explicar a semelhança entre os tempos das atividades. Alterações nos tempos despendidos nas atividades de alimentação e ruminação têm sido freqüentemente observadas em trabalhos nos quais as dietas experimentais apresentaram variações nos teores de fibra (Beauchemin, 1991; Carvalho et al., 2006b). Silva et al. (2005a) observaram redução nos tempos de alimentação e ruminação em novilhas leiteiras alimentadas com dietas contendo bagaço de mandioca. Segundo esses autores, a redução do teor de fibra nas dietas com a inclusão de bagaço de mandioca foi o fator responsável por esses comportamentos. Carvalho et al. (2006b), no entanto, ao fornecerem níveis crescentes de FDN da forragem em dietas para cabras leiteiras, verificaram aumento nos tempos de alimentação e ruminação e diminuição no tempo de ócio.

Neste experimento, a semelhança entre os tempos de alimentação, ruminação e ócio entre os níveis de farelo de cacau confirma os resultados obtidos por Carvalho et al. (2006a), que estudaram a inclusão de farelo de cacau e torta de dendê em dietas para ovinos machos inteiros da raça Santa Inês e também verificaram comportamento semelhante entre os tempos de alimentação, ruminação e ócio.

O número de bolos ruminados por dia ( $\mathrm{n}$ \%/dia) apresentou comportamento quadrático, de modo que o valor máximo foi estimado em 882,31 bolos para o nível de 14,76\% de farelo

Tabela 2 - Consumos de matéria seca (MS) e de fibra em detergente neutro (FDN) em 24 horas, tempo (mim) despendido em alimentação, ruminação $(\mathrm{mim})$ e ócio $(\mathrm{mim})$, número de bolos ruminados por dia e tempo (seg) médio gasto em mastigações por bolo em ovinos alimentados com dietas contendo farelo de cacau

\begin{tabular}{|c|c|c|c|c|c|c|c|c|c|}
\hline \multirow[t]{2}{*}{ Item } & \multicolumn{4}{|c|}{ Farelo de cacau (\%) } & \multirow[b]{2}{*}{$\mathrm{CV}^{1}$} & \multicolumn{2}{|c|}{ Efeito } & \multirow[t]{2}{*}{ Regressão } & \multirow[t]{2}{*}{$\mathrm{R}^{2}$} \\
\hline & 0 & 10 & 20 & 30 & & $\mathrm{~L}$ & $\mathrm{Q}$ & & \\
\hline $\begin{array}{l}\text { Consumo de MS em } \\
24 \text { horas (kg) }\end{array}$ & 1,40 & 1,43 & 1,34 & 1,35 & 9,2 & ns & ns & $\hat{\mathrm{Y}}=1,38$ & - \\
\hline $\begin{array}{l}\text { Consumo de FDN } \\
\text { em } 24 \text { horas (kg) }\end{array}$ & 0,61 & 0,64 & 0,56 & 0,57 & 9,3 & ns & ns & $\hat{\mathrm{Y}}=0,60$ & - \\
\hline Alimentação (min) & 295 & 337,5 & 300,0 & 275 & 12,8 & ns & ns & $\hat{\mathrm{Y}}=301,88$ & - \\
\hline Ruminação (min) & 430 & 487,5 & 487,5 & 462,5 & 14,8 & ns & ns & $\hat{\mathrm{Y}}=466,88$ & - \\
\hline Ócio (min) & 715 & 615 & 652,5 & 702,5 & 13,3 & ns & ns & $\hat{\mathrm{Y}}=671,25$ & - \\
\hline Bolos ruminados ( $\mathrm{n}$ ㅇ/dia) & 646,2 & 914,6 & 799,9 & 668,2 & 15,6 & ns & 0,005 & $\hat{Y}=664,532+29,5169 X-1,00017 X^{2}$ & 0,86 \\
\hline $\begin{array}{l}\text { Tempo de mastigações/ } \\
\text { bolo (seg) }\end{array}$ & 40,0 & 32,0 & 37,0 & 42,0 & 11,4 & ns & 0,005 & $\hat{Y}=39,10-0,8275 X+0,03125 X^{2}$ & 0,84 \\
\hline
\end{tabular}

1 Efeito: $L$ = linear; $Q$ = quadrática. 
de cacau. O tempo médio gasto em mastigações por bolo ruminado (seg) também apresentou comportamento quadrático e o valor mínimo foi de 33,62 para o nível de 13,24\% de farelo de cacau (Tabela 2). Entretanto, a redução do número de bolos ruminados por dia com a adição de $14,76 \%$ de farelo de cacau foi compensada pelo aumento no tempo de mastigações por bolo (Tabela 3), o que explica a elevação do número de mastigações merícicas por bolo ruminado, que aumentou $(\mathrm{P}<0,05)$ linearmente, com acréscimo de 0,28 mastigações para cada unidade de farelo de cacau adicionada ao concentrado. Por outro lado, o número de mastigações por dia apresentou comportamento quadrático, com valor máximo de 42.818,4 mastigações diárias para o nível de $16,9 \%$ de farelo de cacau, o que pode estar relacionado à redução do número de bolos ruminados por dia, que também decresceu com a adição de nível semelhante de farelo (14,76\%).

As eficiências de alimentação e ruminação (g MS e FDN/hora) não foram significativas. Os valores médios de eficiência em alimentação foram 278,2 e 119,9 g e os de eficiência de ruminação 181,6 e 78,4 g, respectivamente, para as frações MS e FDN. Do mesmo modo, Carvalho et al. (2004) avaliaram níveis de 0, 15 e 30\% de farelo de cacau ou torta de dendê em dietas para cabras leiteiras e também não notaram diferença significativa para a eficiência em alimentação, contudo, observaram menor eficiência de ruminação para a dieta com maior nível de farelo de cacau, decorrente dos menores consumos de MS e FDN. Neste trabalho, os consumos de MS e FDN (kg/dia) foram semelhantes entre as dietas e apresentaram valores médios, respectivamente, de 1,38 e $0,60 \mathrm{~kg}$ (Tabela 2), o que justifica os resultados obtidos para as eficiências, que são diretamente relacionadas ao consumo expresso em $\mathrm{kg} / \mathrm{dia}$.

Segundo Van Soest (1994), o teor de fibra e a forma física da dieta são os principais fatores que afetam o tempo de ruminação. Como as dietas apresentaram teores de FDN bastantes semelhantes, a eficiência de ruminação não foi afetada, pois, segundo Dado \& Allen (1995), o número de períodos de ruminação aumenta de acordo com o teor de fibra da dieta, o que reflete a necessidade de processamento da digesta ruminal para elevar a eficiência digestiva, fato não observado neste trabalho.

Os períodos de refeição e ruminação (no/dia) não foram significativos $(\mathrm{P}>0,10)$ e seus valores médios foram de 13,6 e 20,2, respectivamente (Tabela 3). Carvalho et al. (2006a) avaliaram a inclusão de 0 e $16 \%$ de farelo de cacau ou torta de dendê na dieta total de ovinos e também não verificaram diferenças nos períodos das atividades de alimentação, ruminação e ócio. Nesta pesquisa, o tempo despendido por período (min) também não foi significativo $(\mathrm{P}>0,05)$ e refletiu a semelhança nos tempos de alimentação, ruminação e ócio (min/dia) e no número de período de cada atividade (n⿳⺈/dia) entre os níveis de farelo testados, indicando que o fornecimento de farelo de cacau não afeta a discretização das séries temporais em ovinos nas condições de alimentação deste experimento (Tabela 4).

Tabela 3 - Eficiência de alimentação (g MS e FDN/hora), eficiência de ruminação (g MS e FDN/hora), mastigações merícicas e número de períodos de refeição, ruminação e ócio ( $n$ \%/dia) em ovinos alimentados com dietas contendo farelo de cacau

\begin{tabular}{|c|c|c|c|c|c|c|c|c|c|}
\hline \multirow[t]{2}{*}{ Item } & & \multicolumn{3}{|c|}{ Farelo de cacau (\%) } & & \multicolumn{2}{|c|}{ Efeito } & \multirow[t]{2}{*}{ Regressão } & \multirow[t]{2}{*}{$\mathrm{R}^{2}$} \\
\hline & 0 & 10 & 20 & 30 & $\mathrm{CV}^{1}$ & $\mathrm{~L}$ & $\mathrm{Q}$ & & \\
\hline
\end{tabular}

Eficiência de alimentação (g MS e FDN/hora)

\begin{tabular}{|c|c|c|c|c|c|c|c|c|}
\hline MS & 285,8 & 259,3 & 272,0 & 295,8 & 11,2 & NS & NS & $\hat{\mathrm{Y}}=278,2$ \\
\hline FDN & 124,5 & 115,1 & 114,3 & 125,8 & 11,0 & NS & NS & $\hat{\mathrm{Y}}=119,9$ \\
\hline
\end{tabular}

Eficiência de ruminação (g MS e FDN/hora)

\begin{tabular}{|c|c|c|c|c|c|c|c|c|c|}
\hline MS & 204,9 & 180,0 & 165,6 & 175,9 & 18,1 & NS & NS & $\hat{\mathrm{Y}}=181,6$ & - \\
\hline FDN & 89,3 & 79,9 & 69,6 & 74,8 & 18,4 & NS & NS & $\hat{\mathrm{Y}}=78,4$ & - \\
\hline \multicolumn{10}{|c|}{ Mastigação merícica } \\
\hline №/bolo & 46,3 & 46,5 & 50,3 & 54,3 & 6,1 & 0,0012 & NS & $\hat{\mathrm{Y}}=45,1500+0,2775 \mathrm{X}$ & 0,90 \\
\hline №/dia & $29.717,3$ & $42.810,6$ & $40.338,6$ & $36.143,3$ & 17,4 & NS & 0,0207 & $\hat{Y}=30409,3+1464,71 X-43,2215 X^{2}$ & 0,90 \\
\hline
\end{tabular}

Período de refeição, ruminação e ócio (no/dia)

\begin{tabular}{lllllllll} 
Refeição & 13,7 & 14,6 & 13,9 & 12,2 & 16,1 & NS & NS & $\hat{Y}=13,6$ \\
Ruminação & 17,8 & 22,1 & 20,4 & 20,4 & 11,1 & NS & NS & $\hat{Y}=20,2$ \\
Ócio & 26,0 & 28,0 & 28,0 & 29,0 & 9,4 & 0,0903 & NS & $\hat{Y}=26,0750+0,10750 X$ \\
\hline
\end{tabular}

${ }^{1}$ Efeito: $\mathrm{L}=$ linear; $\mathrm{Q}=$ quadrática. 
Os consumos de MS e FDN expressos em kg/refeição e em minutos/kg também não foram significativos ( $\mathrm{P}>0,05)$ (Tabela 4). Diferenças significativas nessas variáveis seriam esperadas caso o tempo de alimentação, o número de refeições diárias e o consumo de MS e FDN fossem influenciados pela adição de farelo de cacau na dieta dos animais.

A atividade de ruminação, expressa em min/kg de MS e FDN, não foi afetada pela adição de farelo de cacau na dieta (Tabela 5). Embora o teor de FDN nas dietas tenha variado com a inclusão do farelo, a diferença máxima entre os teores desta fração (7,34 unidades percentuais) nas dietas com 0 e $30 \%$ de adição não foram suficientes para provocar alterações nas atividades de ruminação. Um fator que pode ter favorecido a ausência de efeito sobre a ruminação é o pequeno tamanho das partículas do farelo de cacau, semelhantes ao dos alimentos concentrados milho moído e farelo de soja.

O tempo de mastigação total em min/kg de MS e FDN não foram influenciados $(\mathrm{P}>0,05)$ pelos níveis de farelo de cacau, provavelmente em virtude da semelhança entre os tempos despendidos em alimentação e ruminação entre as dietas, uma vez que o tempo total é obtido pela soma do tempo gasto em alimentação e ruminação em 24 horas.

Tabela 4 - Consumos de MS e de FDN por refeição $(\mathrm{kg})$, consumos de MS e de FDN $(\mathrm{min} / \mathrm{kg})$ e tempo gasto por período de refeição, ruminação e ócio (min) em ovinos alimentados com dietas contendo farelo de cacau

\begin{tabular}{|c|c|c|c|c|c|c|c|c|}
\hline \multirow[t]{2}{*}{ Item } & \multicolumn{4}{|c|}{ Farelo de cacau (\%) } & \multirow[b]{2}{*}{$\mathrm{CV}^{1}$} & \multicolumn{2}{|c|}{ Efeito } & \multirow[t]{2}{*}{ Regressão } \\
\hline & 0 & 10 & 20 & 30 & & $\mathrm{~L}$ & $\mathrm{Q}$ & \\
\hline \multicolumn{9}{|c|}{ Consumo de MS e FDN/refeição (kg) } \\
\hline MS & 0,105 & 0,098 & 0,099 & 0,113 & 16,5 & NS & NS & $\hat{\mathrm{Y}}=0,104$ \\
\hline FDN & 0,046 & 0,044 & 0,042 & 0,048 & 16,3 & NS & NS & $\hat{\mathrm{Y}}=0,045$ \\
\hline \multicolumn{9}{|c|}{ Consumo de MS e FDN (min/kg) } \\
\hline MS & 210,7 & 236,0 & 223,9 & 203,7 & 12,7 & NS & NS & $\hat{\mathrm{Y}}=218,6$ \\
\hline FDN & 483,6 & 531,5 & 532,9 & 479,1 & 12,5 & NS & NS & $\hat{\mathrm{Y}}=506,8$ \\
\hline
\end{tabular}

Período de refeição, ruminação e ócio (min)

\begin{tabular}{|c|c|c|c|c|c|c|c|c|}
\hline Refeição & 22,1 & 23,3 & 22,1 & 22,8 & 18,5 & NS & NS & $\hat{\mathrm{Y}}=22,6$ \\
\hline Ruminação & 24,0 & 22,3 & 24,2 & 23,1 & 18,3 & NS & NS & $\hat{\mathrm{Y}}=23,4$ \\
\hline Ócio & 28,2 & 22,0 & 23,4 & 24,4 & 16,7 & NS & NS & $\hat{\mathrm{Y}}=24,5$ \\
\hline
\end{tabular}

${ }^{1}$ Efeito: $\mathrm{L}=$ linear; $\mathrm{Q}=$ quadrática.

Tabela 5 - Tempo gasto em ruminação e mastigação total em ovinos alimentados com dietas contendo farelo de cacau

\begin{tabular}{|c|c|c|c|c|c|c|c|c|c|}
\hline \multirow[t]{2}{*}{ Item } & \multicolumn{4}{|c|}{ Farelo de cacau (\%) } & & \multicolumn{2}{|c|}{ Efeito } & \multirow[t]{2}{*}{ Regressão } & \multirow[t]{2}{*}{$\mathrm{R}^{2}$} \\
\hline & 0 & 10 & 20 & 30 & $\mathrm{CV}^{1}$ & $\mathrm{~L}$ & $\mathrm{Q}$ & & \\
\hline
\end{tabular}

Ruminação (g de MS e FDN/bolo)

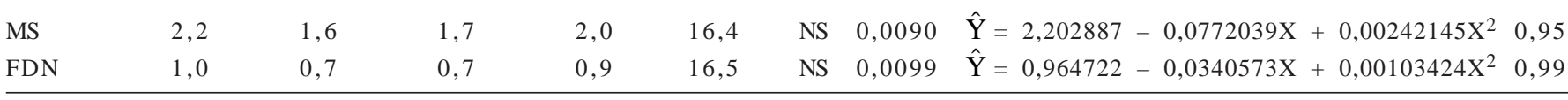

Ruminação (min/kg de MS e FDN)

\begin{tabular}{lccccccccc} 
MS & 307,1 & 340,9 & 363,8 & 342,6 & 14,6 & NS & NS & $\hat{Y}=338,6$ & - \\
FDN & 704,9 & 767,72 & 865,9 & 805,8 & 14,4 & NS & NS & $\hat{Y}=786,1$ & - \\
\hline
\end{tabular}

Mastigação total (min/kg de MS e FDN)

$\begin{array}{lcccccccc}\text { MS } & 517,9 & 576,9 & 587,7 & 546,3 & 11,4 & \text { NS } & \text { NS } & \hat{Y}=557,2 \\ \text { FDN } & 1.188,52 & 1.299,21 & 1.398,76 & 1.284,84 & 10,2 & \text { NS } & \text { NS } & \hat{Y}=1292,8\end{array}$

1 Efeito: $L$ = linear; $Q$ = quadrática. 


\section{Conclusões}

A utilização de farelo de cacau em níveis de até 15\% da dieta total de ovinos Santa Inês afeta alguns parâmetros do comportamento alimentar, mas não altera de forma significativa o comportamento ingestivo e não compromete o desempenho produtivo dos animais.

\section{Literatura Citada}

BEAUCHEMIN, K.A. Effects of dietary neutral fiber concentration and alfafa hay quality on chewing, rumen function, and milk production of dairy cows. Journal of Dairy Science, v.74, n.9, p.3140-3151, 1991.

BÜRGER, P.J.; PEREIRA, J.C.; QUEIROZ, A.C. et al. Comportamento ingestivo em bezerros holandeses alimentados com dietas contendo diferentes níveis de concentrado. Revista Brasileira de Zootecnia, v.29, n.1, p.236-242, 2000.

CARVALHO, G.G.P.; PIRES, A.J.V.; SILVA, F.F. et al. Comportamento ingestivo de cabras leiteiras alimentadas com farelo de cacau ou torta de dendê. Pesquisa Agropecuária Brasileira, v.39, n.9, p.919-925, 2004.

CARVALHO, G.G.P.; PIRES, A.J.V.; SILVA, R.R. et al. Comportamento ingestivo de ovinos alimentados com dietas compostas de silagem de capim-elefante amonizada ou não e subprodutos agroindustriais. Revista Brasileira de Zootecnia, v.35, n.4, p.1805-1812, 2006a.

CARVALHO, S.; RODRIGUES, M.T.; BRANCO, R.H. et al. Comportamento ingestivo de cabras Alpinas em lactação alimentadas com dietas contendo diferentes níveis de fibra em detergente neutro proveniente da forragem. Revista Brasileira de Zootecnia, v.35, n.2, p.562-568, 2006b.

CARVALHO, G.G.P.; PIRES, A.J.V.; SILVA, H.G.O. et al. Aspectos metodológicos do comportamento ingestivo de cabras lactantes alimentadas com farelo de cacau e torta de dendê. Revista Brasileira de Zootecnia, v.36, n.1, p.103-110, 2007.

CUNHA NETO, P.A. Capim-elefante (Pennisetum purpureum Schum.) amonizado, farelo de cacau (Theobroma cacao L.) e torta de dendê (Elaeis guineensis, Jacq) na alimentação de ovinos. Itapetinga: Universidade Estadual do Sudoeste da Bahia, 2004. 42p. Dissertação (Mestrado em Zootecnia) Universidade Estadual do Sudoeste da Bahia, 2004.
DADO, R.G.; ALLEN, M.S. Intake limitation, feeding behavior, and rumen function of cows challenged with rumen fill from dietary fiber or inert bulk. Journal of Dairy Science, v.78, n.1, p.118-133, 1995.

FISCHER, V. Efeito do fotoperíodo, da pressão de pastejo e da dieta sobre o comportamento ingestivo de ruminantes. Porto Alegre: Universidade Federal do Rio Grande do Sul, 1996. 243p. Tese (Doutorado em Zootecnia) - Universidade Federal do Rio Grande do Sul, 1996.

MIRANDA, L.F.; QUEIROZ, A.C.; VALADARES FILHO, S.C. et al. Comportamento ingestivo de novilhas leiteiras alimentadas com dietas à base de cana-de-açúcar. Revista Brasileira de Zootecnia, v.28, n.3, p.614-620, 1999.

PIRES, A.J.V., VIEIRA, V.F., SILVA, F.F. et al. Níveis de farelo de cacau (Theobroma cacao) na alimentação de bovinos. Revista Electrónica da Veterinária, v.6, n.2, p.1-10, 2005.

PIRES, A.J.V.; CARVALHO JR., J.N.; SILVA, F.F. et al. Farelo de cacau (Theobroma cacao) na alimentação de ovinos. Revista Ceres, v.26, n.286, p.33-46, 2004.

POLLI, V.A.; RESTLE, J.; SENNA, D.B. et al. Aspectos relativos à ruminação de bovinos e bubalinos em regime de confinamento. Revista Brasileira de Zootecnia, v.25, n.5, p.987-993, 1996.

SILVA, D.J.; QUEIROZ, A.C. Análise de alimentos: métodos químicos e biológicos. Viçosa, MG: Editora UFV, 2002. 235p.

SILVA, H.G.O.; PIRES, A.J.V.; SILVA, F.F. et al. Farelo de cacau (Theobroma cocoa L.) e torta de dendê (Elaeis guineensis, Jacq) na alimentação de cabras em lactação: consumo e produção de leite. Revista Brasileira de Zootecnia, v.34, n.5, p.17901798, out. 2005b.

SILVA, R.R.; MAGALHÃES, A.F.; CARVALHO, G.G.P. de. et al. Comportamento ingestivo de novilhas mestiças de holandês suplementadas em pastejo de Brachiaria decumbens. Aspectos metodológicos. Revista Electrónica de Veterinaria, v.5, n.10, p.1-7, 2004.

SILVA, R.R.; SILVA, F.F.; CARVALHO, G.G.P. et al. Comportamento ingestivo de novilhas mestiças de holandês x zebu confinadas. Archivos de Zootecnia, v.54, n.205, p.75-85, 2005a.

SNIFFEN, C.J.; O'CONNOR, D.J.; Van SOEST, P.J. et al. A net carbohydrate and protein system for evaluating cattle diets: carbohydrate and protein availability. Journal of Animal Science, v.70, n.12, p.3562-3577, 1992.

UNIVERSIDADE FEDERAL DE VIÇOSA - UFV. SAEG - Sistema de análises estatísticas e genéticas. Viçosa, MG: 2000. (CD-ROM).

Van SOEST, P.J. Nutritional ecology of the ruminant. 2.ed. Ithaca: Cornell University Press, 1994. 476p. 